\title{
Is there a relationship between whole grain intake and biomarkers of nutritional status?
}

\author{
A.P. Nugent ${ }^{1}$, N.F.C. Devlin ${ }^{1}$, J. Walton ${ }^{2}$, A. Flynn ${ }^{2}$ and B.A. McNulty ${ }^{1}$ \\ ${ }^{1}$ UCD Institute of Food and Health, University College Dublin, Belfield, Dublin 4, Ireland and ${ }^{2}$ School of Food and \\ Nutritional Sciences, University College Cork, Cork, Ireland
}

Previously, consumption of wholegrain foods has been related to a more nutrient dense diet in Irish adults ${ }^{(1)}$. This analysis investigated any associations between eating wholegrain foods and biomarkers of nutritional status using data from the National Adult Nutrition Survey (2008-2010) (www.iuna.net) ${ }^{(2)}$. A 4-day semi-weighed food diary recorded food intake data of 1500 adults and wholegrain intake was identified at food and brand level ${ }^{(1)}$. Markers of nutritional status were measured by ELISA (serum 25 $(\mathrm{OH})$ vitamin $\mathrm{D})$, microbiological assay (red blood cell (RBC) and serum folate), fluorescence polarization immunoassay (plasma homocysteine; Hcy), HPLC (plasma pyridoxal-5'-phosphate; PLP, vitamin B6), erythrocyte glutathione reductase activation coefficient (EGRAC; riboflavin), Beckman Coulter Counter (Haemoglobin; Hb) or a RX Daytona automated analyser (serum ferritin, Total Iron Binding Capacity; TIBC). After the exclusion of under-reporters, non-consumers of whole grain were identified and tertiles of intake calculated for consumers.

\begin{tabular}{|c|c|c|c|c|c|c|c|c|c|}
\hline & \multicolumn{9}{|c|}{ Tertiles of mean daily whole grain intakes* } \\
\hline & \multicolumn{2}{|c|}{$\begin{array}{l}\text { Non-consumers } \\
\mathrm{n}=94\end{array}$} & \multicolumn{2}{|l|}{$\begin{array}{l}\text { Tertile } 1 \\
\mathrm{n}=298\end{array}$} & \multicolumn{2}{|l|}{$\begin{array}{l}\text { Tertile } 2 \\
\mathrm{n}=313\end{array}$} & \multicolumn{2}{|l|}{$\begin{array}{l}\text { Tertile } 3 \\
\mathrm{n}=346\end{array}$} & \multirow[t]{2}{*}{$P$ value } \\
\hline & Median & IQR & Median & IQR & Median & IQR & Median & IQR & \\
\hline Wholegrain (g/d) & $0^{\mathrm{a}}$ & 0 & $8 \cdot 6^{\mathrm{b}}$ & $10 \cdot 1$ & $31 \cdot 3^{\mathrm{c}}$ & $11 \cdot 8$ & $68 \cdot 3^{\mathrm{d}}$ & $38 \cdot 1$ & $<0.001$ \\
\hline Serum ferritin (ng/ml) & $95 \cdot 8$ & $101 \cdot 7$ & $87 \cdot 4$ & $109 \cdot 4$ & $78 \cdot 9$ & $100 \cdot 7$ & $93 \cdot 0$ & 121.9 & 0.901 \\
\hline TIBC $(\mu \mathrm{mol} / \mathrm{L})$ & $59 \cdot 7$ & $11 \cdot 4$ & $59 \cdot 9$ & $15 \cdot 4$ & $60 \cdot 9$ & $12 \cdot 7$ & $59 \cdot 0$ & $11 \cdot 0$ & $0 \cdot 502$ \\
\hline Haemoglobin (g/dL) & $14 \cdot 1$ & $2 \cdot 7$ & $14 \cdot 3$ & $2 \cdot 1$ & $13 \cdot 9$ & 1.8 & $14 \cdot 3$ & 1.8 & 0.587 \\
\hline Serum folate $(\mathrm{nmol} / \mathrm{L})$ & $17 \cdot 8^{\mathrm{a}}$ & $14 \cdot 6$ & $20 \cdot 9^{\mathrm{a}}$ & $17 \cdot 0$ & $28 \cdot 3^{\mathrm{b}}$ & $24 \cdot 2$ & $31.9^{\mathrm{b}}$ & $27 \cdot 8$ & $<0.001$ \\
\hline $\mathrm{RBC}$ folate $(\mathrm{nmol} / \mathrm{L})$ & $790 \cdot 4^{\mathrm{a}}$ & $387 \cdot 9$ & $799 \cdot 8^{\mathrm{a}}$ & $424 \cdot 0$ & $908.7^{\mathrm{b}}$ & 558 & $1019 \cdot 5^{\mathrm{b}}$ & $584 \cdot 4$ & $<0.001$ \\
\hline Serum B12 (nmol/L) & $269 \cdot 3^{\mathrm{a}}$ & $141 \cdot 9$ & $273 \cdot 6^{\mathrm{a}}$ & $155 \cdot 3$ & $302 \cdot 1^{\mathrm{b}}$ & $137 \cdot 2$ & $329 \cdot 4^{\mathrm{b}}$ & $169 \cdot 8$ & $<0.001$ \\
\hline EGRAC & $1.4^{\mathrm{a}}$ & $0 \cdot 3$ & $1 \cdot 4^{\mathrm{a}}$ & $0 \cdot 2$ & $1 \cdot 3^{\mathrm{b}}$ & $0 \cdot 2$ & $1 \cdot 3^{\mathrm{b}}$ & $0 \cdot 2$ & $<0.001$ \\
\hline Plasma PLP (nmol/L) & $77 \cdot 1^{\mathrm{a}}$ & $60 \cdot 4$ & $79 \cdot 4^{\mathrm{a}}$ & $62 \cdot 0$ & $89 \cdot 0^{\mathrm{b}}$ & $69 \cdot 5$ & $93 \cdot 5^{\mathrm{b}}$ & $59 \cdot 5$ & $<0.001$ \\
\hline Plasma Hcy (nmol/L) & $13 \cdot 0^{\mathrm{a}}$ & $4 \cdot 8$ & $12 \cdot 1^{\mathrm{a}}$ & $3 \cdot 9$ & $11 \cdot 4^{\mathrm{b}}$ & $3 \cdot 1$ & $11 \cdot 3^{\mathrm{b}}$ & $3 \cdot 8$ & $<0.001$ \\
\hline Serum $25(\mathrm{OH}) \mathrm{D}(\mathrm{nmol} / \mathrm{L})$ & $48 \cdot 1$ & $40 \cdot 1$ & $56 \cdot 6$ & $28 \cdot 8$ & $56 \cdot 4$ & $33 \cdot 7$ & $59 \cdot 8$ & $35 \cdot 7$ & $0 \cdot 249$ \\
\hline
\end{tabular}

Higher dietary wholegrain intakes were associated with improvements in B-vitamin and homocysteine status but not in biomarkers of iron or vitamin D intake. Across the consumption groups, there were no changes $(\mathrm{P}>0.05)$ in dietary intakes $(\mathrm{mg}$ or $\mu \mathrm{g} / 10 \mathrm{MJ} / \mathrm{day})$ of iron, vitamin D or of any of the B vitamins studied. Although correlations were weak, dietary intakes of wholegrain containing breads, ready to eat breakfast cereals and cooked breakfast cereals were more strongly $(\mathrm{P}<0.05)$ related with $\mathrm{B}$-vitamin and homocysteine status than other wholegrain containing foods. Further research will identify whether benefits of eating wholegrain containing foods exist beyond B-vitamin status.

The project was funded by the Irish Department of Agriculture, Food and the Marine under the Nutridata Project 13 F 542; $2014-2016$.

1. Devlin NFC, McNulty BA, Walton et al. (2014) Proc Nutr Soc. Summer Meeting, Glasgow.

2. IUNA (2011) National Adult Nutrition Survey Summary Report. http://www.iuna.net. March 2015 